\title{
ELVES reconstruction and characterization at the Pierre Auger Observatory
}

\author{
Roberto Mussa $^{* a}$ for the Pierre Auger Collaboration ${ }^{b \dagger}$ \\ a Istituto Nazionale di Fisica Nucleare, Sezione di Torino, Italy \\ ${ }^{b}$ Observatorio Pierre Auger, Av.San Martín Norte 304, 5613 Malargüe, Argentina \\ E-mail: auger_spokespersons@fnal.gov \\ Full author list: http://www.auger.org/archive/authors_icrc_2019.html
}

\begin{abstract}
Since 2013, the four sites of the Fluorescence Detector (FD) of the Pierre Auger Observatory have been recording transient luminous events called ELVES with a dedicated trigger. These light emissions are correlated to distant lightning strikes. The length of recorded traces has been increased from $100 \mu \mathrm{s}$ (2013) to $300 \mu \mathrm{s}$ (2014-16) and $900 \mu \mathrm{s}$ (2017-present), to calculate better the total energy of the UV light emitted at the base of the ionosphere and its angular distribution above the lightning strike. The reconstruction of the time evolution of the light front allows an accurate determination of the lightning bolt location, within a few kilometers, as confirmed by the comparison with ground-based lightning detection networks. The time resolution of the FD applied to the observation of ELVES is a novel approach to probe fundamental physics in thunderstorms such as their charge distribution and lightning initiation processes. Argentina has been flagged as one of the most active regions on Earth for the most extreme lightning strikes, which highlight the serendipitous opportunity the Auger Observatory is offering to the geophysics community.
\end{abstract}

36th International Cosmic Ray Conference -ICRC2019-

July 24th - August 1st, 2019

Madison, WI, U.S.A.

\footnotetext{
* Speaker.

${ }^{\dagger}$ For collaboration list see PoS(ICRC2019)1177
} 


\section{Introduction}

Besides its main activity, the study of the ultra-high-energy cosmic rays, the Pierre Auger Observatory has started a program of cosmo-geophysics studies, which exploits some of the unique features of its detectors. Since 2013, during the hours of operation of the Fluorescence Detector (FD), a dedicated data stream is devoted to the study of ELVES. This phenomenon, predicted in the early 1990s [1] and discovered one year later [2], are transient luminous events occuring at an altitude of about $90 \mathrm{~km}$ when an intense electromagnetic pulse (EMP) emitted during the development of lightning reaches the base of the ionosphere. For an observer at the ground, with a camera capable to record images at the speed of more than one million frames per second, ELVES appear as rapidly expanding rings, starting from a point at mid-distance between source and observer, progressively increasing in brightness as the front drifts towards the horizon, to form a projected smile into the camera. With longer integration time (e.g. more than $5 \mathrm{~ms}$ per frame) the ELVES will appear like a flat ring in the sky, approximately centered above the vertical of the lightning. The 24 FD telescopes of the Auger Observatory [3], each with an $11 \mathrm{~m}^{2}$ mirror, 440 PMTs with high photon sensitivity in the close UV band ( $\lambda=300$ to $420 \mathrm{~nm}$ ), and a time resolution of $100 \mathrm{~ns}$, are ideally suited for such type of observations. These studies have required a series of modifications to the online selection of the events, based on a three-stage trigger described in the next section.

\section{Triggering ELVES with the Auger FD}

The first level trigger operates at the pixel level with an adjustable threshold that keeps the PMT trigger rate constant at $100 \mathrm{~Hz}$. The second level trigger (SLT) searches for track segments made of at least five adjacent pixels which passed the first level trigger. The third level trigger (TLT) was originally designed to efficiently veto events due to close lightning, featuring a high multiplicity of triggered pixels randomly distributed in time across the camera within a $100 \mu \mathrm{s}$ time window.

After the serendipitous discovery of ELVES in our early data sets [4], the upgrade of the multiplicity-based TLT prevented the further recording of ELVES candidates in the standard data stream. A four-year long study of prescaled SLT events, which yielded 58 new ELVES candidates [5], facilitated the design of a TLT algorithm which was able to both reject close lightning and accept the ELVES events, when the direct flash from the causative lightning is below the horizon. The new TLT is performing a check on the angular evolution of the light front around the first triggered pixel. The pixels in the rows and the columns adjacent to the first triggered pixel are checked; their pulse peak time is required to grow continuously with the distance from the first pixel. To account for statistical fluctuations, $20 \%$ of the time increments between two triggered adjacent pixels are allowed to show the wrong slope. As ELVES candidates release a large amount of light in comparison to cosmic rays, the trigger also requires at least one pixel with a pulse amplitude larger than 50 ADC counts.

\section{ELVES data taken from 2013 to 2016}

The ELVES trigger was at first implemented in March 2013 at the FD site of Los Leones, and then extended to all FD sites during the following FD shift. During the first year of commissioning, 

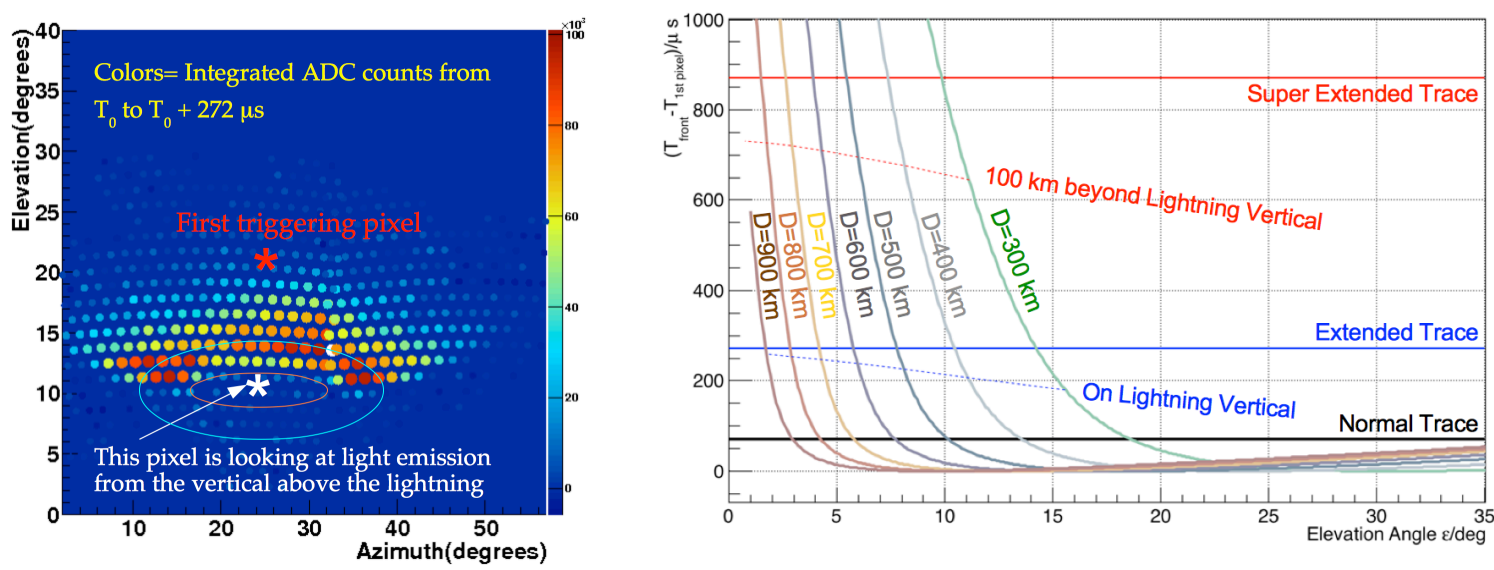

Figure 1: Light distribution after $272 \mu \mathrm{s}$ (left); time evolution of the light front in the central column (right) for ELVES caused by lightning at distances from 300 to $900 \mathrm{~km}$ (green to brown curves); the solid horizontal lines show the trace length in 2013 (black), 2014-16 (blue), 2017-present (red). The dashed oblique lines show when the light front reaches the vertical above the lightning and the region of maximum intensity, about $100 \mathrm{~km}$ beyond the vertical.

we learned that the standard FD traces (which are $100 \mu \mathrm{s} \mathrm{long)} \mathrm{did} \mathrm{not} \mathrm{allow} \mathrm{us} \mathrm{to} \mathrm{see} \mathrm{the} \mathrm{light}$ emitted from the region of the ionosphere located vertically above the lightning source. Therefore, the FD readout scheme was modified in order to acquire three consecutive frames for the ELVES triggers (Extended Readout). This change enables the study of the angular distribution of light emission above the lightning, which contains information on the dynamics of this process. It must be stressed that the time width of the large EMP generating the ELVES is of the order of $10^{-5} \mathrm{~s}$, four to five orders of magnitude shorter than the full development of the lightning bolt, and two orders of magnitude shorter than the time needed for the light wave to propagate across the base of the ionosphere. In addition, the size of the central gap is related to the electrons' speed in the return stroke of the lightning [6].

The 2014-2016 ELVES dataset was taken with a maximum trace length of $300 \mu \mathrm{s}$. A typical example of an event taken with the extended readout is shown in Fig.1, where the distribution of light, integrated over the first $272 \mu$ s after a $28 \mu$ s buffer used for pedestal calculation, is shown. The vertical above the lightning is represented by a white star; the red star indicates the first triggered pixel, while the red ellipse indicates the region where the light emission dies down. Modelling the EMP emission from the lightning source as an ideal vertical dipole, we expect an azimuthal symmetry of the light emission around the vertical above the lightning. Partial reconstruction of the amount of light emitted by a fraction of the region of peak intensity would allow an estimate of the total light emission in the UV band. During the three-year period, we collected about $2.3 \cdot 10^{3}$ ELVES candidate triggers: $80 \%$ are mono (i.e. seen from only one FD site), $17 \%$ are stereo (simultaneously seen from two sites), and $2.2 \%$ are triplet (seen from three sites). Further quality checks were used to increase the purity of the sample.

The multiple eye capability of the Observatory has been exploited to have a first estimate of the trigger efficiency of each FD. After correcting for on-time of operation [7] of each site, and accounting for the geographically not uniform distribution of lightning strikes in the field of view, 


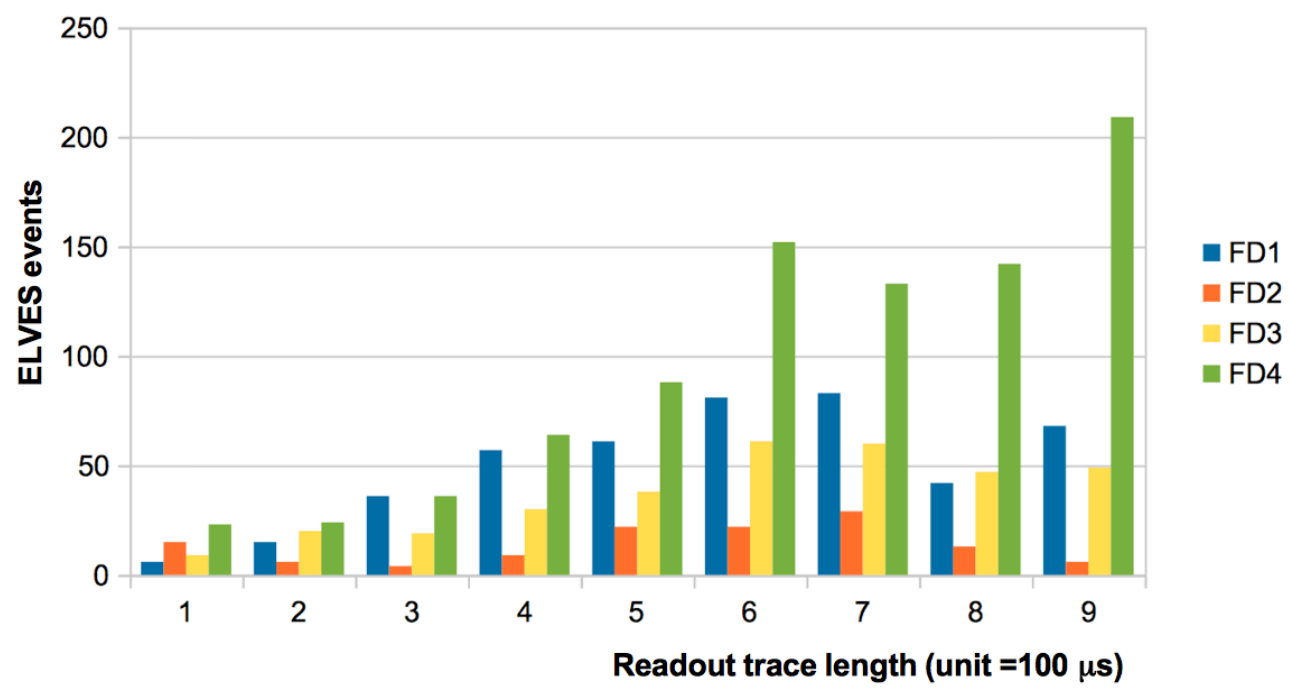

Figure 2: Distribution of the trace lengths for ELVES triggers for each FD site, in units of $100 \mu \mathrm{s}$, for the period 2017-2018. The FD4 site (Coihueco) sees more ELVES because the majority of thunderstorms occur in its field of view.

we could estimate a single FD efficiency $\varepsilon_{F D}=35 \pm 8 \%$.

The study of the ELVES events taken in the period 2014-2016 has shown that a more complex variety of emission patterns has to be considered. The estimate of the total UV emission, to be compared with the energy of the original EMP pulse, requires the measurement of the intensity of light through the full region of maximum emission, outlined by the cyan ellipse on the left panel of Fig.1. In order to explore the light emission from angular regions at lower elevation angles, it was necessary to largely increase the integration time. In the right panel of Fig.1, the difference between the time of arrival of the light front on the central column of the ELVES and the time of the first triggered pixel, is shown, as a function of the pixel elevation, for ELVES produced by lightning at distances from 300 to $900 \mathrm{~km}$.

Since January 2017, the ELVES trace length has been extended to a maximum of $900 \mu \mathrm{s}$ (Super Extended Readout scheme) in order to observe the peak intensity region of most events through the whole azimuth range around the lightning generating the ELVES. In Fig.2, the distribution of the size of the traces recorded in the four FD sites from 2017 to 2018 is shown in units of $100 \mu \mathrm{s}$, which is the standard for cosmic ray triggers.

\section{Reconstruction of lightning location}

The geometrical reconstruction of the light front propagation aims to a precise time and space location of lightning which caused the ELVES, and to a good measurement of the angular distribution of the light emission from the base of the ionosphere, located at an average altitude $H_{E M}=92$ $\mathrm{km}$. The lightning location algorithm is based on two very simple assumptions: (a) the emission of the EMP at the lightning can be considered point-like in space-time from the FD observer, and (b) the de-excitation time scale of the base of the ionosphere is negligible with respect to the prop- 


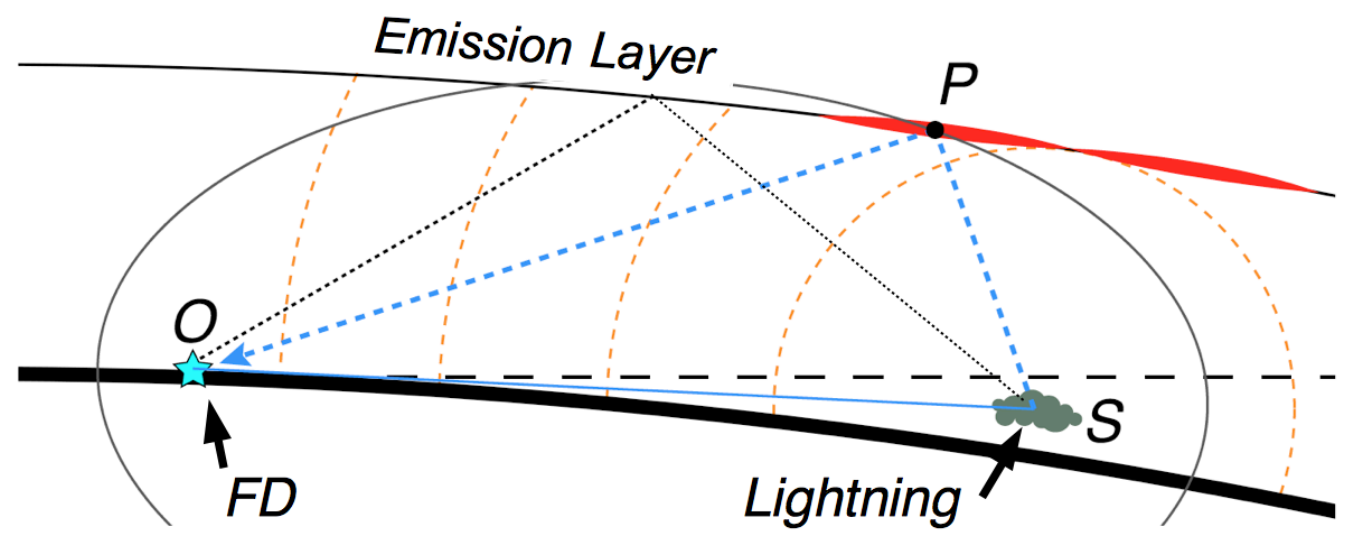

Figure 3: ELVES reconstruction geometry.

agation time. With these two simple assumptions we can conclude that, at any given instant, the emitting surface at the base of the ionosphere can be calculated as the intersection of an ellipsoid, whose focal points are the source $\mathrm{S}$ and the observer $\mathrm{O}$, with a sphere whose radius is $R_{\text {earth }}+H_{E M}$. The azimuthal expansion of this curve is expected to be symmetric with respect to the vertical, while the front going towards the FD is expected to move faster than that going in the opposite direction.

In the first step of the reconstruction, we fit the ADC trace of each FD pixel to one or more asymmetric gaussians. If less than ten pixels feature a double peak, the reconstruction proceeds with a precise calculation of the time and location of the lightning strike. When ten or more pixels have a double peak, the reconstruction requires a more complex algorithm, which is briefly described in the following sections.

The reconstruction of the lightning location is performed as a two-step process:

1. a set of polynomial fits is performed on the pulse times $T_{i}$ of each row and column, to obtain a first rough estimate of the lightning longitude and latitude; the results are inputs to the second fit;

2. we perform a second fit to minimize the $\chi^{2}=\Sigma_{i=1}^{N}\left(T_{i}-\Delta T_{0}-\overline{O P S}_{i} / c\right)^{2} / \sigma_{T, i}^{2}$ where $N$ is the number of pixels contributing to the minimisation, $\Delta T_{0}$ is the time lag between the emission of the EMP from the source and the observation of the first light at the FD diaphragm, and $\overline{O P S}_{i}$ is the sum of the distances $\overline{S P}$ (from the source to the emission layer) and $\overline{P O}$ (from the emission layer, assumed to have zero thickness and altitude $H_{E M}=92 \mathrm{~km}$, to the FD); the lightning is assumed to be at sea level.

In this way, the location of the lightning source for almost 1600 events is reconstructed. These have then been compared with the lightning recorded by the World Wide Lightning Location Network (WWLLN, [8]) within $1500 \mathrm{~km}$ from the centre of the Observatory. We found that $\simeq 70 \%$ of our events are correlated with a bolt of lightning detected by the WWLLN within $5 \mathrm{~ms}$. 

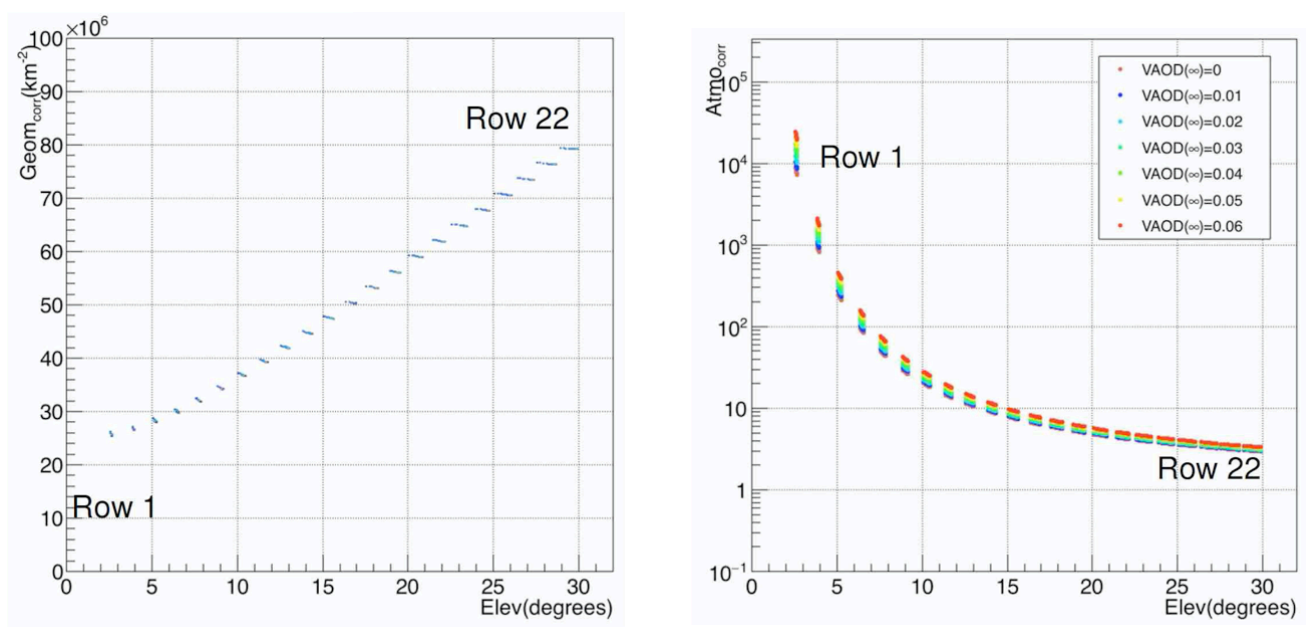

Figure 4: Geometric corrections at the centre of each pixel (left), atmospheric corrections with total VAOD ranging from 0 to 0.06 (right) .

\section{Reconstruction of the angular distribution of the light emitted at the base of the ionosphere}

After having located the lightning source, in order to calculate the total amount of photons radiated by the ELVES, it is necessary to transform the amount of light observed in each pixel, $P_{i}(F D)$, to the photon surface density $\Phi_{i}=P_{i}(F D) * C_{i}($ geom $) * C_{i}($ atmo $)$ at the base of ionosphere. The geometric correction

$$
C_{i}(\text { geom })=\overline{O P}^{2} /\left(A_{\text {mirror }} A_{E M}\right)
$$

accounts for the solid angle subtended by the FD diaphragm, and the size of the emitting surface at the base of the ionosphere $A_{E M}$, constructed projecting the six vertices of each pixel to an altitude of $92 \mathrm{~km}$.

The atmospheric correction:

$$
C_{i}(\text { atmo })=\exp \left((\operatorname{VMOD}(\infty)+\operatorname{VAOD}(\infty)) * \operatorname{AM}\left(\theta_{i}\right)\right)
$$

accounts for the absorption of light through the atmosphere, using the total vertical optical depths of molecules, $\operatorname{VMOD}(\infty)$, and aerosols, $\operatorname{VAOD}(\infty)$, through the atmosphere, measured by the instruments on site [9]. The airmass $\operatorname{AM}(\theta)$ is calculated using the formula in ref.[10].

The corrections (shown in Fig.4 for the center of each pixel) are strongly dependent on the row number and weakly dependent on the column number. After applying these corrections, in the rare case of a perfectly vertical current source, the total amount of light emitted from the base of the ionosphere can be easily extrapolated. Further work is needed to model tilted dipoles or more complex emission patterns.

\section{Classification of Multiple ELVES}

About one fifth of the ELVES identified at the Auger Observatory are composed of more than one distinct flash of light. When more than ten pixels show more than one pulse in the traces, the 


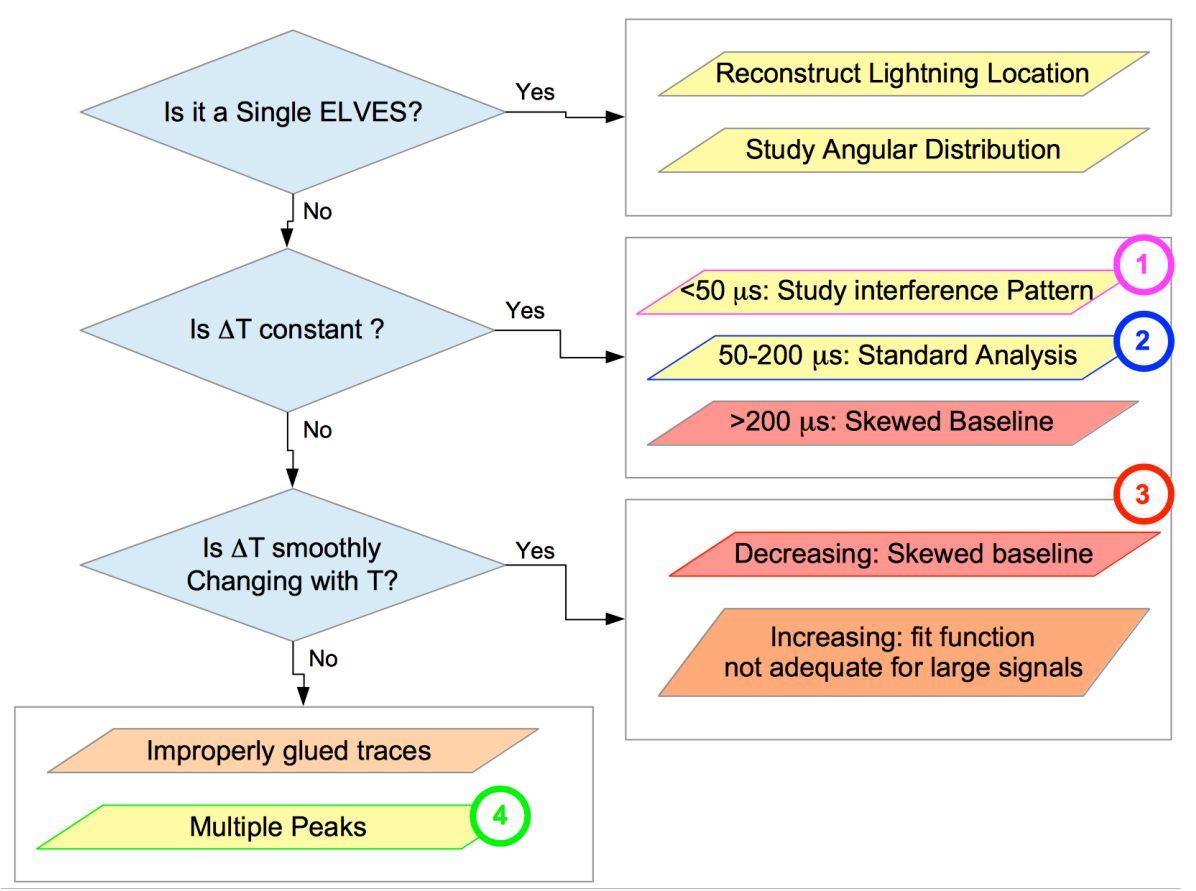

Figure 5: Flowchart of ELVES analysis chains.

ELVES is labeled as multiple and a different type of analysis (summarised in Fig.5) is performed. The first step consists in plotting the time difference between any two pulses, as shown in Fig.6, where one event for each of the four categories found by the current analysis is depicted.

The first two categories are characterized by a constant $\Delta T$ : when it is very short $(<50 \mu \mathrm{s})$ it may be explained as the interference pattern produced by the EMP emitted by an intracloud lightning when a fraction of the pulse is bouncing off the ground. If the gap is larger than $50 \mu \mathrm{s}$, the events are likely to be related to the initial breakdown stages of the lightning, or to a more complex profile of the return stroke. The third category consists of events with a $\Delta T$ linearly decreasing with pulse time, which appear as single ELVES overlapped to another class of light transient, maybe a halo [11], with a much longer risetime. The fourth group, showing abruptly changing $\Delta T$, is related to ELVES with more than two peaks. This pattern has been proposed as a possible signature of production of terrestrial gamma-ray flashes [12]. A simple linear fit of $\Delta T$ vs the time $T$ of the first peak allows differentiating these four categories, as shown in the right part of Fig.6. The first three categories account for $80 \%$ (respectively $11 \%, 57 \%, 22 \%$ ) of the total number of events. The remaining events, with a large positive slope, are very likely to be triple or quadruple ELVES and deserve further analysis.

\section{Conclusions and future prospects}

Since 2013, the Auger Observatory has been taking data with a special trigger devoted to the study of ELVES. The time resolution of our FD gives us unique opportunities for the study of these phenomena. We changed the length of the trace readout in two steps: (a) since 2014, we sampled $300 \mu \mathrm{s}$ in order to see the light emission from the vertical of the causative lightning; (b) since 2017, 

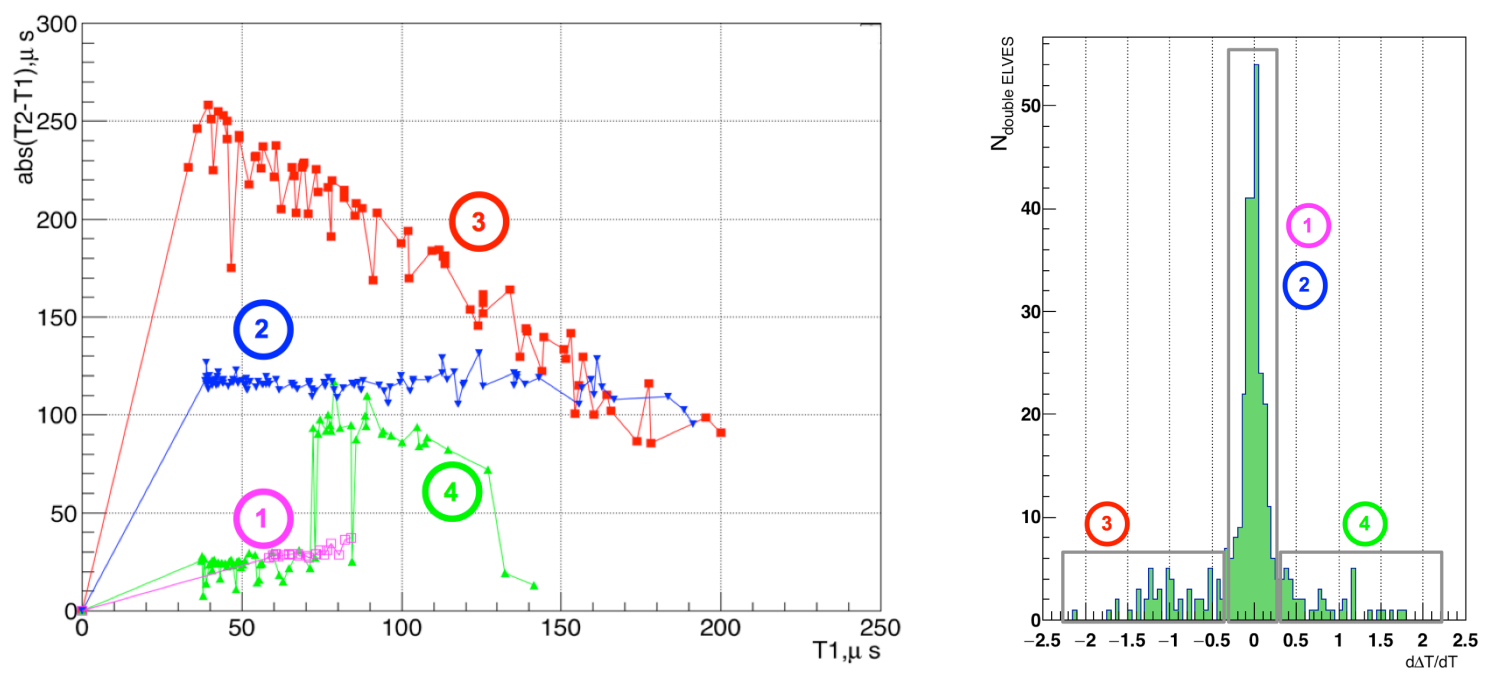

Figure 6: Time difference between two pulses vs time of the first pulse in multiple ELVES (left). Distribution of the slopes of the time between the first and the second pulse in multiple ELVES (right): the numbers indicate the categories shown in the left plot and described in the text.

we read up to $900 \mu \mathrm{s}$, to study the angular distribution of the entire region of maximum emission around the lightning.

In most cases, we have learned that simple models are not able to describe the rich variety of ELVES events, and larger statistics are required to fully understand the variety of features shown by such interesting phenomena.

For future analyses, we envisage further upgrades of the TLT selection criteria to increase the selection efficiency. We are also planning to enhance our lightning detection network to gain more insights on the mechanisms leading to the production of multiple ELVES.

\section{References}

[1] U. S. Inan, T. F. Bell, J. V. Rodriguez, Geophys. Res. Lett. 18, (1991) 705

[2] W. L. Boeck et al., Geophys. Res. Lett. 19, (1992) 99

[3] J. Abraham et al. [Pierre Auger Collaboration], Nucl. Instrum. Meth. A 620 (2010) 227.

[4] R. Mussa et al. [Pierre Auger Collaboration], Eur. Phys. J. Plus 127 (2012) 94.

[5] A. Aab et al. [Pierre Auger Collaboration], arXiv:1307.5059 [astro-ph.HE].

[6] P. R. Blaes et al., Geophys. Res. Lett.41 (2014) 9182.

[7] P. Abreu et al. [Pierre Auger Collaboration], Astropart. Phys. 34 (2011) 368.

[8] http://wwlln.net.

[9] J. Abraham et al. [Pierre Auger Collaboration], Astropart. Phys. 33 (2010) 108.

[10] F. Kasten and A. T. Young, Applied Optics 28 (1989) 4735.

[11] C.P. Barrington-Leigh et al., J. Geophys. Res. 106 (2001) 1141.

[12] N. Liu et al., J. Geophys. Res. 122 (2017) 10563. 